\title{
Those Troublesome but Never Tiresome Triphasic Waves
}

Can. J. Neurol. Sci. 2006; 33: 125

Electroencephalographically speaking, triphasic waves (TWs) and generalized spike waves (GSWs), as in nonconvulsive generalized status epilepticus, are biologically distinct, but can closely resemble one another on the scalprecorded EEG. ${ }^{1}$ As numerous authors have pointed out, TWs are characteristic of metabolic encephalopathies. ${ }^{2-5}$ However, they have also been described in sepsis, meningitis and cortical degenerative diseases. ${ }^{6-9}$ Many of these conditions can also cause seizures, so even within diagnostic categories the electrographic differentiation is important. This often causes consternation in the EEGer, even if he/she resorts to the clinical context to help differentiate TWs and GSWs. The study by Boulanger and colleagues goes farther than any other clinical neurophysiological study in providing practical guidelines to the clinical EEGer. ${ }^{10}$ There are helpful observations on the morphological characteristics of each entity: seizures are associated with presence of polyspikes, while triphasic waves differ from spike-waves in the prominence of the second (positive) component in TWs, a front-to back or back-to-front phase shift in TWs, disappearance in sleep and response to stimuli (an original and potentially very useful point). It should be remembered that triphasic waves do not occur in children, so the differentiation is not an issue in pediatric EEG.

There are some limitations to the study by Boulanger et al. ${ }^{10}$ It is retrospective and some relevant information may be lacking. It uses traditional differentiating EEG criteria that have never been subjected to pathophysiological investigations for confirmation. Such an approach is almost tautological. Nonetheless, their approach of having the records read blindedly, then correlating with the clinical problem, making careful note of various features of the electrographic behaviour (effects of drugs, sleep and stimulation) as well as the morphology and distribution, adds credibility and makes further useful observations. The inter-rater and clinical correlations are impressive, and the settling of differences by going with the original classification is so infrequent as to be negligible.

Although the neurophysiological mechanisms underlying spike-waves are generally accepted, TWs have never been adequately explained. They appear to be "waves" rather than nonstationarities the way spikes are; they can be produced by combining different frequencies with a sine wave generator
(Blume WT, personal communication). Nunez has studied "traveling" and "standing" waves mathematically. ${ }^{11}$ The classical phase shift of TWs is likely due to a wave or waves traveling in an inhomogenous medium, probably with a number of interacting spatially separated "oscillators" that may be purely cortical or perhaps cortical-thalamic. It would be wonderful if a physiologist could confirm the above in an elegant experiment. We clinical neurophysiologists must await such a development with hopeful expectation. Not only will this be of intellectual interest, but it may provide even more differentiating guidelines or new technical approaches.

\section{G. Bryan Young London, Ontario}

\section{REFERENCES}

1. Kaplan PW. The EEG in metabolic encephalopathy and coma. J Clin Neurophysiol. 2005;21:307-18.

2. Bickford RG, Butt HR. Hepatic coma: the electroencephalographic pattern. J Clin Invest .1995;34:790-99.

3. Karnaze DS, Bickford RG. Triphasic waves: a reassessment of their significance. Electroenceph Clin Neurophysiol. 1984;57:193-8.

4. Bauer G, Noedermeyer E. Acute convulsions. Clin Electroenceph. $1979 ; 10: 127-44$

5. Lawrence J. Hirsch, Richard P. Brenner, Frank W. Drislane. The ACNS Subcommittee on Research Terminology for Continuous EEG Monitoring: Proposed Standardized Terminology for Rhythmic and Periodic EEG Patterns Encountered in Critically Ill Patients, J Clin Neurophysiol. 2005;22:128-35.

6. Young GB, Bolton CF, Austin TW, Archibald YM, Wells GA. The encephalopathy associated with septic illness. Clin Invest Med. 1990;13:297-304.

7. Blatt I, Brenner RP. Triphasic waves in a psychiatric population: a retrospective study. J Clin Neurophysiol. 1996;13:324-9.

8. Sundaram MB, Siemens P. Triphasic waves in Mollaret's meningitis. J Neurol Neurosurg Psychiatry. 1986;49:331

9. Sundaram MBM, Blume WT. Triphasic waves: clinical correlates and morphology. Can J Neurol Sci. 1987;14:136-40.

10. Boulanger J-M, Deacon C, Lécuyer D, Gosselin S, Reiher J. Triphasic waves versus nonconvulsive status epilepticus: EEG distinction. Can J Neurol Sci 2006; 33:175-80.

11. Nunez PL. Electric Fields of the Brain Oxford:Oxford University Press; 1981; p. 315-348. 